\title{
Transport-controlled reaction rates under local non-equilibrium conditions
}

\author{
Xavier Sanchez-Vila, ${ }^{1}$ Marco Dentz, ${ }^{1}$ and Leonardo David Donado ${ }^{1,2}$ \\ Received 19 January 2007; revised 17 March 2007; accepted 30 April 2007; published 31 May 2007.
}

[1] Chemical reactions are driven by non-equilibrium and can be fully described by the spatio-temporal distribution of the reaction rate. We present an analytical approach for the computation of reaction rates under local non-equilibrium conditions for a precipitation/dissolution problem. We derive an original non-linear partial differential equation for the reaction rate $r$ and present a series expansion of $r$ for large Damköhler numbers, i.e., fast local scale reactions. The impact of local scale non-equilibrium conditions on the transport-controlled reaction rate is studied for reactive transport in a column. Citation: Sanchez-Vila, X., M. Dentz, and L. D. Donado (2007), Transport-controlled reaction rates under local non-equilibrium conditions, Geophys. Res. Lett., 34, L10404, doi:10.1029/2007GL029410.

\section{Introduction}

[2] Chemical reactions in aquatic systems are driven by non-equilibrium conditions. When at a given point the concentrations of the reacting species do not satisfy the law of mass action, the system reacts by changing these concentrations, driving the system towards equilibrium. In reactive transport problems, reactions are driven by (1) local non-equilibrium due to deviations of the locally uniform species concentrations from the law of mass action and (2) global non-equilibrium due to transport-controlled mixing, which leads to spatial variations in the aqueous species concentrations.

[3] Solute transport and mixing are governed by advection, diffusion and hydrodynamic dispersion. These processes are linear in the species concentrations. Chemical interactions induce non-linearities, since the reaction rates, incorporated as sink/source terms in the global mass balances, are non-linear in the species concentrations. The local scale reaction rates are modeled by inferring the dynamics determined in a well-mixed reactor while assuming complete mixing of the species locally.

[4] The chemical evolution of a multispecies system can be fully described in terms of reaction rates. In a well stirred environment (complete mixing assumed), the reaction rate is homogeneous in space due to the uniform species concentrations, and solely varies in time. Its temporal evolution reflects the reaction dynamics and describes the relaxation of the system towards equilibrium. In a reactive transport problem, reaction rates are a function of space and time

\footnotetext{
${ }^{1}$ Hydrogeology Group, Department of Geotechnical Engineering and Geosciences, Technical University of Catalonia, Barcelona, Spain.

${ }^{2}$ School of Civil Engineering, Industrial University of Santander, Bucaramanga, Colombia.
}

Copyright 2007 by the American Geophysical Union. 0094-8276/07/2007GL029410 because the species concentrations vary spatio-temporally. The reaction rate provides a map of where reactions occur in the medium. As such, reaction rates contain valuable information about the transport-controlled global reaction dynamics. Thus, in order to properly address and understand the dynamics of multicomponent reactive transport, it is necessary to have a methodology at hand to compute the reaction rates at any location in space and time.

[5] Because of its mathematical complexity, the solution of multicomponent reactive transport problems in general, and the computation of reaction rates in particular, have been mostly tackled using numerical approaches. A few studies deal with analytical solutions to simplified reactive transport problems, such as homogeneous bimolecular reactions [Ham et al., 2004], biodegradation in natural attenuation problems [Liedl et al., 2005; Cirpka et al., 2006], and heterogeneous or homogeneous reactions involving multiple aqueous species and minerals [De Simoni et al., 2005, 2007]. In all these cases the geochemical problems are characterized by local instantaneous equilibrium. Kechagia et al. [2002] studied the upscaling of fast chemical reactions from the pore to the Darcy scale using volume-averaging techniques and discuss the validity of the continuum approach for fast local scale reactions.

[6] Local scale equilibrium can be assumed to hold if the reaction time $\tau_{r}$ is small compared to a typical dispersion time scale $\tau_{D}$. The dispersion scale is compared to the reaction time scale $\tau_{r}$ by the non-dimensional Damköhler number $D a$, defined as [e.g., Knapp, 1989],

$$
D a=\frac{\tau_{D}}{\tau_{r}} .
$$

Note that dispersion is the relevant mass transfer mechanism in the context of the mixing-limited reactions under consideration here. Advective transport has the effect of a mere translation of a fluid element in space.

[7] Under the assumption of an instantaneous local chemical reaction, i.e., $D a \rightarrow \infty$, De Simoni et al. [2005] presented a methodology for the derivation of the spatiotemporal distribution of the reaction rate. The resulting expression is the product of two terms: (1) a mixingcontrolled contribution, which depends on the local dispersion tensor; and (2) a speciation term, containing the details of the local chemical reaction.

[8] For local non-equilibrium conditions (i.e. finite Damköhler numbers), the species concentrations cannot be decoupled from the reaction rates, complicating the mathematical problem. So, it is not possible to derive an explicit expression for the reaction rate.

[9] Figure 1 illustrates the problem for a simple geochemical system involving precipitation of a mineral caused 
by the reaction of two aqueous species. In the case of instantaneous equilibrium, only the configurations along the line corresponding to the law of mass action (a hyperbola in Figure 1) are valid configurations. If at some point two waters with different chemical signatures (still both in perfect chemical equilibrium with the mineral) mix and no reaction takes place, the concentrations would be along the dashed line, leading for example to point A (oversaturated with respect to the mineral). Instantaneous reaction would take the concentrations to point B. Instead, if equilibrium is non-instantaneous, any point in the $c_{1}-c_{2}$ space is a valid configuration. If water $W 1$ mixes with water $W 2$, and if diffusion is very fast compared to reaction (small $D a$ ) the path would be $W 1-A-B^{\prime}-B$, along straight lines. Any point along the line is valid, and so it is possible that water in point $B^{\prime}$ mixes with additional $W 2$ water; the resulting mixtures would be now along the new dashed line. If, instead, reaction is fast, then the path would be $W 1-B$, but now along the hyperbola. In such a case, the chemical evolution is a succession of equilibrium states.

[10] The assumption of instantaneous equilibrium reduces the dimensionality of the problem through the law of mass action. Thus, non-instantaneous reactions lead to a more complex problem in mathematical terms. Reactions and concentrations cannot be decoupled, and no explicit expressions might be obtained. Instead it is possible to

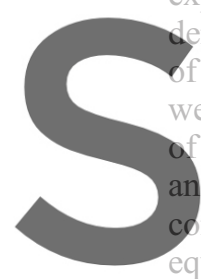
derive a non-linear evolution equation for the distribution reaction rates. For fast loc we derive a perturbation series the inverse Damköhler nuı explicit expression for the quilibrium by De Simoni et al equation
-scale react the reaction
act. In firtstion rate.
tion for
[2005].

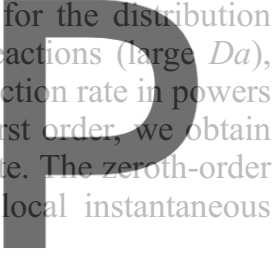

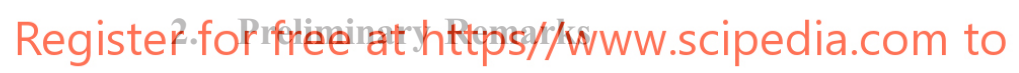

[11] We consider a geochemical problem involving two aqueous species $B_{1}$ and $B_{2}$, and a mineral phase $B_{3}$, which react according to,

$$
B_{1}+B_{2} \rightleftharpoons B_{3}
$$

Without loss of generality, the stoichiometric coefficients of the species are set to one.

[12] Let us first consider reactions in a well mixed reactor. The concentration of the reacting species can be assumed spatially uniform at all times. Mass balance requires for the aqueous species concentrations,

$$
\phi \frac{\mathrm{d} c_{i}}{\mathrm{~d} t}=-r
$$

with $i=1,2$; porosity is denoted by $\phi$. The concentrations of the aqueous species (in molar mass per unit volume of fluid) are denoted by $c_{1}$ and $c_{2}$. Here, the species concentrations depend only on time. For the reaction rate $r(r>0$ indicating precipitation), we employ the model given by, e.g., Knapp [1989], Steefel and Lasaga [1994], Lasaga et al. [1994], and Langmuir [1997],

$$
r=-\tau_{r}^{-1} \sqrt{K}(1-\Omega),
$$

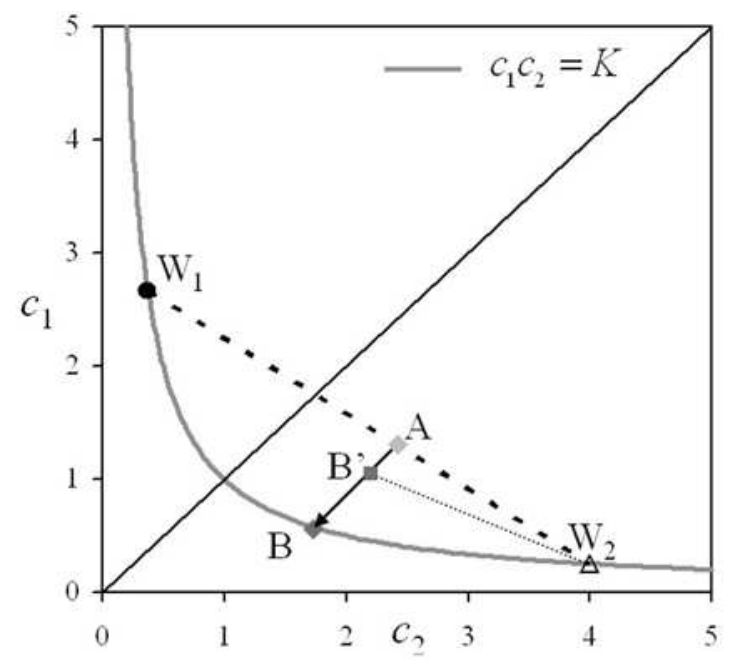

Figure 1. Concentration space map showing the potential values for instantaneous and non-instantaneous equilibrium conditions. In the former, only the values along the hyperbola are valid configurations.

where the effective reaction time $\tau_{r}$ is defined by

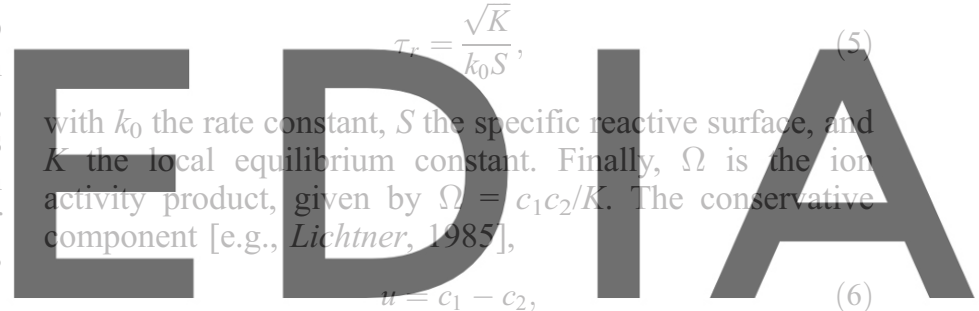

decouples the nonlinear system of equations (3). As d $u / \mathrm{d} t=0$, dondalioaditheomersion with out the watersmark constant. Thus, (3) can be solved straightforwardly for the species concentrations by separation of variables. This yields for the reaction rate the explicit expression,

$$
r=-\tau_{r}^{-1} \sqrt{K}\left(1+\frac{u_{0}^{2}}{4 K}\right)\left[1-\tanh ^{2}\left(\frac{\sqrt{4 K+u_{0}^{2}}}{2 \phi \sqrt{K}} \frac{t}{\tau_{r}}+A\right)\right],
$$

with the constant $A$ given by the initial conditions, $A=\operatorname{arctanh}$ $\left(\frac{c_{1}(0)+c_{2}(0)}{\sqrt{u_{0}^{2}+4 K}}\right)$. In the limiting case of $t \gg \tau_{r}, r$ tends to zero and the system equilibrates. The statement $c_{1} c_{2}=K$ (law of mass action) is, thus, equivalent to $r=0$. For transport-controlled reactions these two statements are not equivalent. We will address this point later.

\section{Transport-Controlled Reaction}

[13] Mass conservation implies that the concentrations $c_{i}(\mathbf{x}, t)$ of the aqueous species $(i=1,2)$ are governed by the following transport equations,

$$
\phi \frac{\partial c_{i}}{\partial t}+\mathbf{q} \cdot \nabla c_{i}-\nabla \cdot \phi \mathbf{D} \nabla c_{i}=-r
$$

The specific discharge is denoted by $\mathbf{q}$, and $\mathbf{D}$ denotes the bulk dispersion tensor. Without loss of generality we set $\phi=1$ 
in the following. The reaction rate $r$ is given by (4), which implicitly assumes that the reacting species are well mixed and their concentrations are uniform on a local support volume $\Delta V_{l}$ characterized by the length scale $l$. Note that the validity of the continuum approach for very fast local scale reactions is in fact subject of discussion. Here, we assume the existence of a local support scale and leave this important question aside. Kechagia et al. [2002] addressed this issue in their paper on the upscaling of reactive transport processes from the pore to the Darcy scale.

[14] We distinguish here two typical transport time scales; the advection time scale $\tau_{a}=l /|\mathbf{q}|$, and the dispersion time scale $\tau_{D}=l^{2} / D$ with $D$ a characteristic dispersion measure (here, for simplicity, we characterize the effect of dispersion by a single time scale, while in general there could be more than one involving the directional dispersion coefficients). The Péclet number $P e=\tau_{D} / \tau_{a}$ guantifies the relative importance of dispersive and advective transport mechanisms. Note that the dispersion scale is the reaction limiting mass transfer mechanism. In the absence of dispersion, no reaction takes place since waters in contact do not mix and are just advected.

[15] We non-dimensionalize (8) by rescaling the spatial coordinates by $l\left(x_{i}^{\prime}=x_{i} / l\right)$, time by the dispersion time scale $\tau_{D}\left(t^{\prime}=t / \tau_{D}\right)$, and concentrations by $\sqrt{K}\left(c_{i}^{\prime}=c_{i} / \sqrt{K}\right)$. This yields
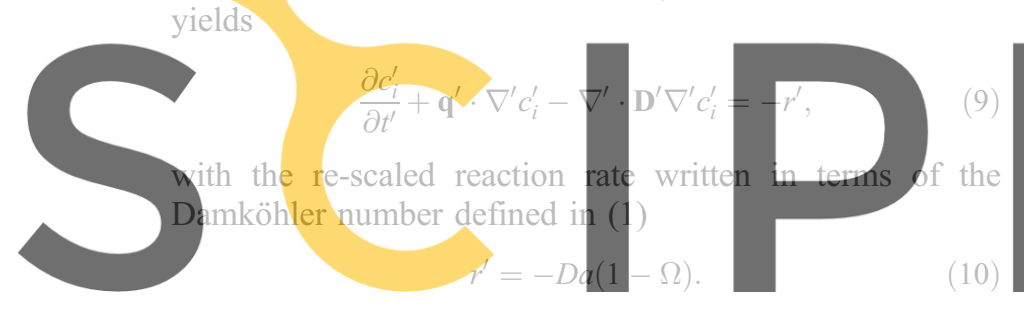

16 The dimensionless discharge and dispersion tensor

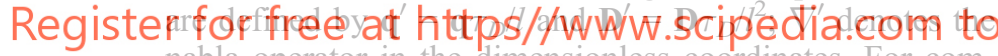
nabla operator in the dimensionless coordinates. For compactness of notation, in the following the primes are omitted.

[17] The independent component (6) is conservative and evolves according to,

$$
\frac{\partial u}{\partial t}+\mathbf{q} \cdot \nabla u-\nabla \cdot \mathbf{D} \nabla u=0
$$

From equations (6) and (10) we obtain explicit expressions for $c_{1}$ and $c_{2}$,

$$
c_{1 / 2}= \pm \frac{u}{2}+\frac{1}{2} \sqrt{u^{2}+4 K(1+\rho)},
$$

where we defined $\rho=r \alpha, \alpha$ being the inverse Damköhler number $\left(\alpha=D a^{-1}\right)$. According to (12), the $c_{i}$ are only functions of the independent components $u$ and $\rho$ (i.e., $c_{i}=$ $c_{i}(u, \rho)$ ). Thus, applying the chain rule to (9), and after some algebra, we obtain the following non-linear partial differential equation for the dimensionless reaction rate $r$,

$$
\begin{gathered}
\alpha\left[\frac{\partial r}{\partial t}+\mathbf{q} \cdot \nabla r-\nabla \cdot(\mathbf{D} \nabla r)\right] \frac{\partial c_{i}}{\partial \rho}-2 \alpha \frac{\partial^{2} c_{i}}{\partial \rho \partial u} \nabla u^{T} \mathbf{D} \nabla r \\
-\alpha^{2} \frac{\partial^{2} c_{i}}{\partial \rho^{2}} \nabla r^{T} \mathbf{D} \nabla r+r=\frac{\partial^{2} c_{i}}{\partial u^{2}} \nabla u^{T} \mathbf{D} \nabla u
\end{gathered}
$$

It turns out that (13) does not depend on $i$, as can be easily seen by inserting (12) into (13); in the following we choose $i=2$.

[18] The solution methodology can be detailed as follows. First, the linear equation (11) is solved for $u$, either numerically or, if possible, analytically. Then $r$ remains the only unknown, and can be obtained by solving (13). Since this equation is non-linear in $r$, the solution must in general be sought numerically. In the case of large Damköhler numbers, $r$ can be expanded into a series in $\alpha=D a^{-1}$, from which an approximate solution for $r$ can be obtained by truncation. This is explored in the next section.

\section{Large Damköhler Numbers}

[19] For $\alpha \ll 1(D a \gg 1)$, the reactive transport system is close to local equilibrium at all points. De Simoni et al. [2005] derived an explicit exact expression for the reaction rate in the case $\alpha=0$, which is equivalent to the condition $c_{1} c_{2}=K$, i.e., the mass action law is fulfilled locally. Their result proves that the $\lim _{\alpha \rightarrow 0} r=r^{(0)}$ exists, which implies that $(1-\Omega) \propto \alpha$ for small $\alpha$ values. Note that for the wellmixed reactor, $c_{1} c_{2}=K$ implies $r=0$, while in a reactive transport scenario, even though local equilibrium is reached instantaneously, the reaction rate remains finite as a consequence of transport-driven changes in the concentration

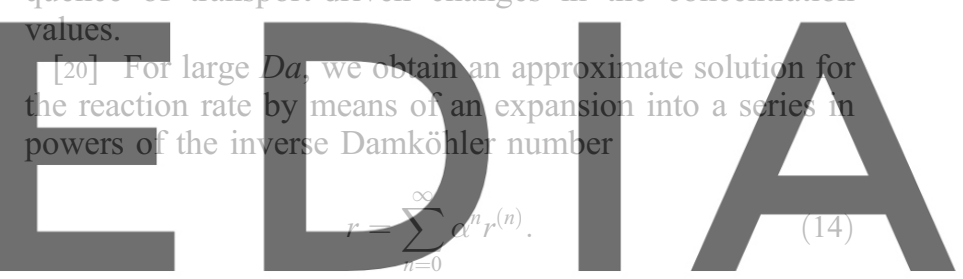

For bounded $r^{(0)}, \rho \ll 1$ for $\alpha \gg 1$. Thus, for small $\alpha$, the

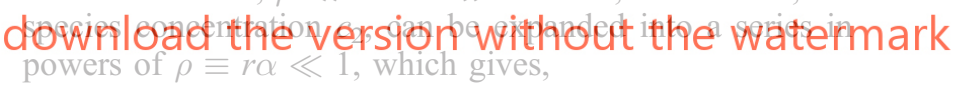

$$
c_{2}=\sum_{n=0}^{\infty} \alpha^{n} r^{n} c_{2}^{(n)}, \quad c_{2}^{(n)}=\left.\frac{1}{n !} \frac{\partial^{n} c_{2}}{\partial \rho^{n}}\right|_{\rho=0}
$$

Inserting (14) into (15), we obtain,

$$
c_{2}=\sum_{k=0}^{\infty} \alpha^{k} \sum_{n=0}^{k} R_{k-n}^{(n)} c_{2}^{(n)}
$$

where the $R_{k}^{(n)}$ are given by

$$
R_{k}^{(n)}=\sum_{m_{1}=0}^{k} r^{\left(k-m_{1}\right)} \sum_{m_{2}=0}^{m_{1}} r^{\left(m_{1}-m_{2}\right)} \ldots \times \sum_{m_{n-1}}^{m_{n-2}} r^{\left(m_{n-2}-m_{n-1}\right)} r^{\left(m_{n-1}\right)}
$$

for $n>1$. In particular, for $n=0, R_{k}^{(0)}=\delta_{k 0}$ and for $n=1$, $R_{k}^{(1)}=r^{(k)}$.

[21] Inserting (14) and (16) into (13) and collecting terms of the order $\alpha^{k}$ yields a recursive relation for $r^{(k)}$. Here we focus on the zeroth and first-order contributions to $r$. The zeroth order term $r^{(0)}$ is given by

$$
r^{(0)}=\frac{\partial^{2} c_{2}^{(0)}}{\partial u^{2}} \nabla u^{T} \mathbf{D} \nabla u
$$




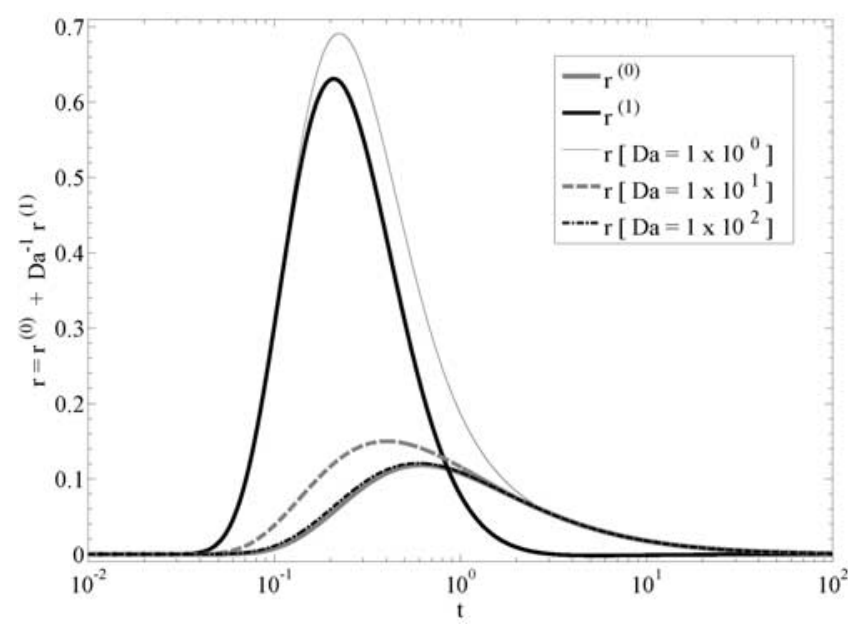

Figure 2. Reaction rate as a function of dimensionless time at the dimensionless position $x=1$ for $P e=10^{-1}$

The resulting expression for $r^{(0)}$ is identical to the one derived by De Simoni et al. [2005] under local instantaneous equilibrium conditions, i.e., in the limit of $D a \rightarrow \infty$ (in this case $\left.c_{2} \equiv c_{2}^{(0)}\right)$. For the first-order contribution $r^{(1)}$, we obtain,

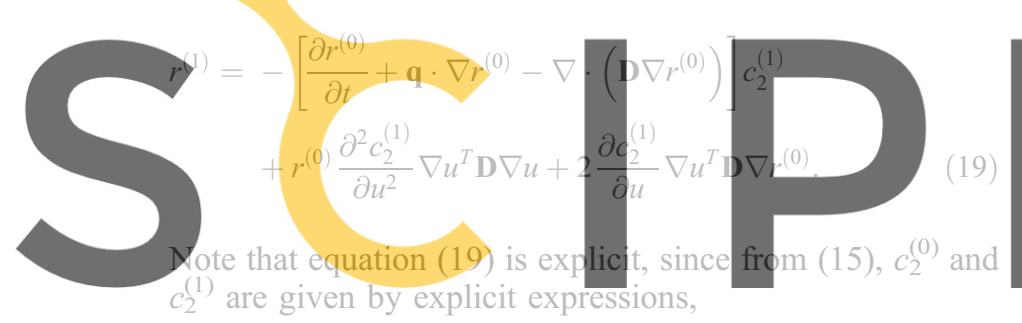

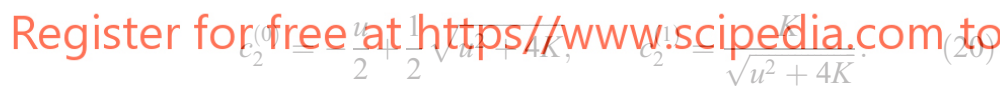

With this same methodology it would also be possible to obtain explicit expressions for higher order terms $r^{(i)}, i>1$.

\section{Case Study}

[22] In the following we study the impact of small deviations from local scale equilibrium on the reaction rate in terms of the first-order correction $r^{(1)}$ to the equilibrium rate $r^{(0)}$. We consider an infinite column filled homogeneously with crushed magnesite $\left(\mathrm{MgCO}_{3}\right)$. The medium is saturated with water which is initially in chemical equilibrium with the mineral. Dissociation of magnesite is described by,

$$
\mathrm{MgCO}_{3} \rightleftharpoons \mathrm{Mg}^{2+}+\mathrm{CO}_{3}^{2-} .
$$

[23] To ensure that the speciation of dissolved inorganic carbon is negligible, we consider the case that the $\mathrm{pH}$ is significantly higher than 10.3 , which is the pKa of the carbonate-bicarbonate system. From Azaroual et al. [2003], a typical reaction time scale for this reaction is of the order of 54 days. Initially, the normalized equilibrium concentrations are $c_{M g^{2+}}^{0} \equiv c_{1}^{0}=0.537$ and $c_{C O_{3}^{2-}}^{0} \equiv c_{2}^{0}=1.858$ (in equilibrium the product of the two normalized concentrations equals 1). A water with different concentrations of each species, in equilibrium with the mineral is injected at $x=0$. The concentrations of the injected water are, $c_{M g^{2+}}^{\text {ext }}=2.148$ and $c_{\mathrm{CO} \mathrm{S}_{3}-}^{\text {ext }}=0.465$. The impact of variations of ionic strength upon $K$ is neglected here.

[24] The initial concentration of the conservative component (6) is $u^{0}=-1.321$, and that of the injected water, $u^{\text {ext }}=1.683$, so that $\Delta u=u^{\text {ext }}-u^{0}=3.004$. Considering continuous injection at $x=0$, the resulting solution of (11) for $u$, reads in dimensionless coordinates

$$
u(x, t)=\frac{\Delta u}{2} \operatorname{erfc}\left(\frac{x-t P e}{2 \sqrt{t}}\right) .
$$

Using (22) in (18) and (19), $r^{(0)}$ and $r^{(1)}$ can be evaluated explicitly.

[25] We consider a dispersion-dominated scenario characterized by the local Péclet number $P e=10^{-1}$. Figure 2 illustrates the temporal evolution of the leading term $r^{(0)}$, the first-order correction $r^{(1)}$ and the first-order approximation $r=r^{(0)}+D a^{-1} r^{(1)}$ at the fixed dimensionless distance $x=1$ for three different values of $D a$.

[26] The non-equilibrium correction $r^{(1)}$ is positive and peaks earlier than the local equilibrium contribution $r^{(0)}$ Note that under local equilibrium conditions the peak of $r^{(0)}$ coincides with the maximum of the gradient of $u(x, t)$, where mixing is most efficient. The local non-equilibrium

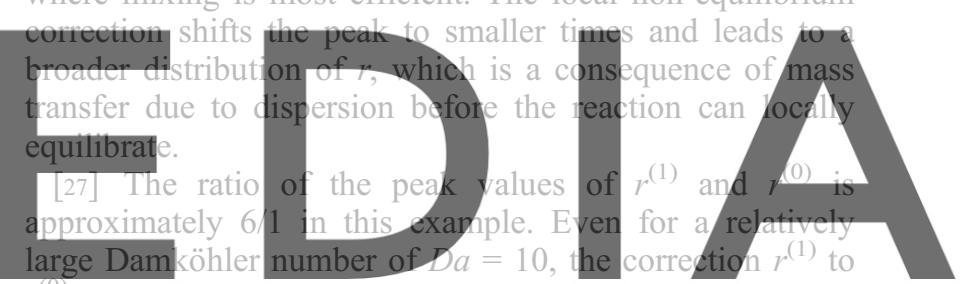

due to local non-equilibrium is sizeable. For illustration, we display the behavior of the first-order correction downaload tha, en wersionatwiłhout dthe envatermark tion is strictly valid only for large $D a$. For a $D a=10^{2}$, the system reaches local equilibrium practically instantaneously and $r^{(0)}$ and the first-order approximation for $r$ are almost indistinguishable.

[28] Figure 3 shows a snapshot of $r^{(0)}, r^{(1)}$ and the firstorder approximation versus distance at a fixed time, again for three different Damköhler numbers. The behavior of $r^{(0)}$ is basically determined by the gradient of the conservative component, see (18), and assumes a maximum where the gradient of $u$ is maximal. The first-order correction shows a very different behavior with two positive peaks and a negative one in between. The peak of the resulting firstorder correction is shifted in the positive $x$-direction as a result of advection. Mass transfer due to dispersion, again, leads to a broader spatial distribution for $r$ rather than for $r_{0}$. For a value of $D a=1$, the shape of the first-order approximation is very different from the one of $r^{(0)}$. It has forward and backward peaks, while in between it can assume negative values, indicating dissolution. However, as mentioned above, the first-order approximation is strictly valid only for large $D a$. Again, for $D a=100, r^{(0)}$ and the firstorder approximation for $r$ are practically indistinguishable.

\section{Conclusions}

[29] We present an analytical approach to study reactive transport under local non-equilibrium conditions. The reac- 


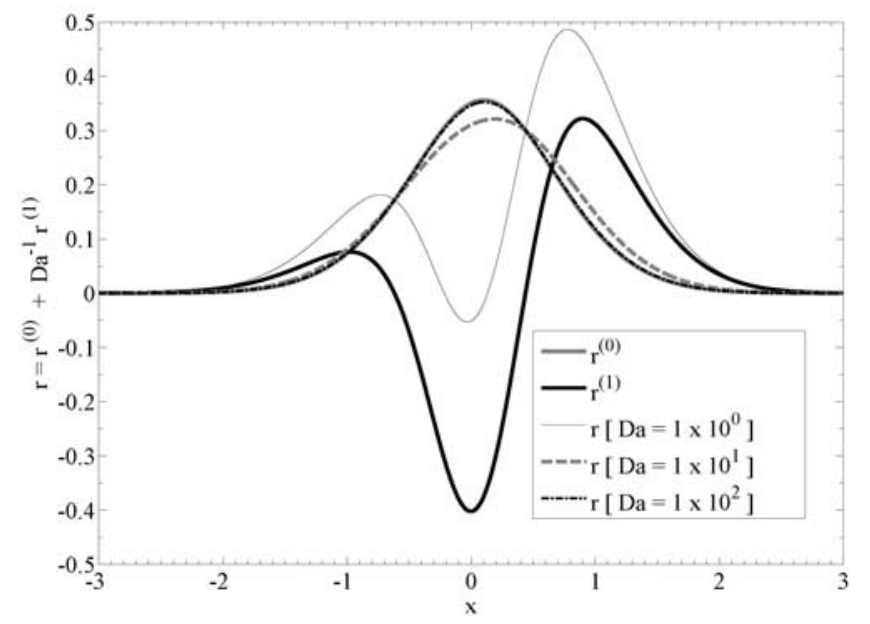

Figure 3. Reaction rate as a function of normalized distance at dimensionless time $t=0.5$ for $P e=10^{-1}$.

tion rate can be used as the main variable characterizing the multispecies transport system. For instantaneously reached chemical equilibrium the reaction rate can be evaluated explicitly in terms of components (linear combinations of the species concentrations behaving conservatively). Under local-scale non-equilibrium conditions the reaction rate can

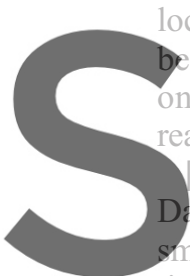
obtained as the solution of the ratio between the reaction times.

30] We use an expansion mall deviations from local e
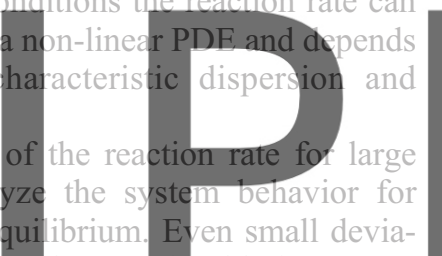
tions from local equilibrium can have a sizeable impact on the shape and magnitude of the reaction rate distribution in

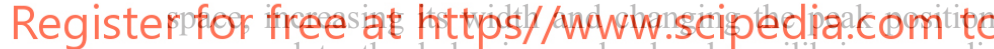
compared to the behavior under local equilibrium conditions. The impact of larger deviations from local equilibrium (typical mass transfer time smaller than the reaction time scale) needs to be analyzed by a full (numerical) solution of the non-linear partial differential equation (13) and comparison to experiments and direct numerical simulations.
[31] Acknowledgments. The authors thank three anonymous reviewers for their comments. The authors gratefully acknowledge the financial support of ENRESA and the EU through the projects FUNMIG and GABARDINE. M. D. acknowledges the support of the Spanish Ministry of Science and Education (MEC) through project MODEST (project CGL-2005-05171) and program "Ramon y Cajal." L. D. acknowledges the support provided by the program AL $\beta$ AN (E03D22383CO).

\section{References}

Azaroual, M., C. Kervévan, M. V. Durance, S. Brochot, and P. Durst (2003), SCALE2000 user's manual, 57 pp., BRGM, Orléans, France.

Cirpka, O. A., A. Olsson, Q. S. Ju, M. A. Rahman, and P. Grathwohl (2006), Determination of transverse dispersion coefficients from reactive plume lengths, Ground Water, 44(2), 212-221.

De Simoni, M., J. Carrera, X. Sánchez-Vila, and A. Guadagnini (2005), A procedure for the solution of multicomponent reactive transport problems, Water Resour. Res., 41, W11410, doi:10.1029/2005WR004056.

De Simoni, M., X. Sanchez-Vila, J. Carrera, and M. W. Saaltink (2007), A mixing ratios-based formulation for multicomponent reactive transport, Water Resour. Res., doi:10.1029/2006WR005256, in press.

Ham, P. A. S., R. J. Schotting, and H. Prommer (2004), Effects of hydrodynamic dispersion on plume lengths for instantaneous bimolecular reactions, Adv. Water Resour, 27(8), 803-813

Kechagia, P. E., I. N. Tsimpanogiannis, Y. C. Yortsos, and P. Lichtner (2002), On the upscaling of reaction-transport processes in porous media with fast or finite kinetics, Chem. Eng. Sci., 57, 2565-2577.

Knapp, R. B. (1989), Spatial and temporal scales of local equilibrium in dynamic fluid-rock systems, Geochim. Cosmochim. Acta, 53, 19551964

Langmuir, D. (1997), Aqueous Environmental Geochemistry, Prentice-Hall, Upper Saddle River, N. J.

Lasaga, A. C., J. M. Soler, J. Ganor, T. E. Burch, and K. L. Nagy (1994), Chemical-weathering rate laws and global geochemical cycles, Geochim.

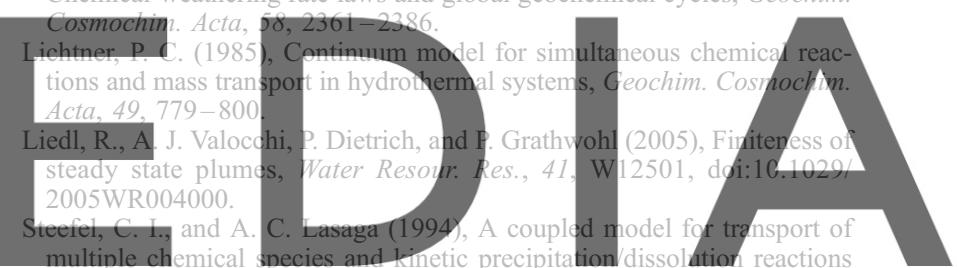

with application to reactive flow in single phase hydrothermal systems, Am. J. Sci. 294, 529-592.

\section{download the version without the watermark}

M. Dentz, L. D. Donado, and X. Sanchez-Vila, Hydrogeology Group, Department of Geotechnical Engineering and Geosciences, Technical University of Catalonia, Jordi Girona 1-3, E-08034 Barcelona, Spain. (marco. dentz@upc.edu; leonardo.david.donado@upc.edu; xavier.sanchez-vila@upc. edu) 